\title{
Fatores que influenciam a textura da carne de novilhos Nelore e cruzados Limousin-Nelore ${ }^{(1)}$
}

\author{
Riana Jordão Barrozo Heinemann ${ }^{(2)}$, Marcos Franke Pinto(3) e Pedro Fernando Romanelli(2)
}

\begin{abstract}
Resumo - O objetivo deste trabalho foi avaliar fatores que influenciam a textura da carne de novilhos Nelore e cruzados Limousin-Nelore. Cinqüenta novilhos, 25 Nelore e 25 Limousin-Nelore, foram aleatoriamente divididos em cinco grupos de 10 animais (cinco de cada grupo genético), para o abate seriado, até 204 dias. Os valores de temperatura e $\mathrm{pH}$ muscular foram monitorados durante 24 horas após o abate. Em seguida, foram medidas a espessura de cobertura de gordura e a área de olho de lombo. O músculo longissimus dorsi retirado foi dividido para avaliação qualitativa do músculo sem maturação e submetido à maturação por 14 dias. A área de olho de lombo foi maior em animais cruzados. Os valores de cobertura de gordura e gordura intramuscular foram semelhantes entre os grupos genéticos. Peso ao abate e teor de gordura afetaram as quedas de $\mathrm{pH}$ e temperatura, mas não resultaram em diferenças na força de cisalhamento. Os animais cruzados apresentaram carne mais macia que os animais Nelore. A maturação causou redução de $30 \%$ na força de cisalhamento e foi, com o fator genético, o parâmetro que mais influenciou a textura da carne.
\end{abstract}

Termos para indexação: carne bovina, maturação, maciez, qualidade.

\section{Factors affecting meat texture from Nellore and crossbreed Limousin-Nellore steers}

\begin{abstract}
The objective of this work was to evaluate factors affecting meat texture from Nellore and crossbreed Limousin-Nellore steers. Fifty steers, 25 Nellore and 25 Limousin-Nellore, were randomly divided in five groups of 10 animals (five from each genetic group) and serially slaughtered during 204 days. Meat temperature and $\mathrm{pH}$ data were monitored during 24 hours post mortem. After this, fat thickness and rib area were measured. Longissimus dorsi muscle was removed to quality evaluation before and after 14 days ageing period. Crossbreed animals rib areas were higher. Fat thickness and marbling values from both genetic groups were similar. Live slaughter weight and fat content affected decrease of $\mathrm{pH}$ and temperature, but didn't result in difference in shear force. Crossbreed animals the most tender meat. Ageing process was responsible for 30\% reduction in shear force, so this was, associated to genetic group, was the principal factor affecting meat texture.
\end{abstract}

Index terms: beef, maturation, tenderness, quality.

(1) Aceito para publicação em 12 de maio de 2003

Extraído da dissertação de mestrado apresentada pelo primeiro autor à Universidade Estadual Paulista (Unesp), São José do Rio Preto, SP.

(2) Unesp, Dep. de Engenharia e Tecnologia de Alimentos, Instituto de Biociências, Letras e Ciências Exatas, Caixa Postal 136, CEP 15054-000 São José do Rio Preto, SP. E-mail: riana_heinemann@ig.com.br, romaneli@eta.ibilce.unesp.br

(3) Unesp, Dep. de Apoio, Produção e Saúde Animal, Fac. de Odontologia de Araçatuba, Caixa Postal 341, CEP 16050-680 Araçatuba, SP. E-mail: mfpinto@fmva.unesp.br

\section{Introdução}

O Brasil possui o maior rebanho bovino comercial do mundo, estimado em 153 milhões de cabeças no último censo agropecuário realizado pelo IBGE em 1996 (IBGE, 2002), tendo ultrapassado 164 milhões de cabeças em 2001 (Anualpec, 2002). O volume de exportação de carne bovina in natura também aumentou 245 mil toneladas, em equivalente carcaça, em 2000, para 479 mil toneladas, em 2002. Esse valor, todavia, é inferior ao volume exportado pelos Esta- 
dos Unidos e Austrália, países com rebanhos comerciais menores. Os Estados Unidos, com um rebanho bovino estimado em 96,5 milhões de cabeças, em 2001, produziu nesse ano quase 12 milhões de toneladas de carne, em equivalente carcaça, enquanto o Brasil produziu cerca de 7 milhões de toneladas (Anualpec, 2002). Esses números mostram a necessidade de aumento da produtividade da bovinocultura brasileira, sempre contemplando os atributos de qualidade, fator essencial para conquista do mercado externo. A introdução de raças européias nos cruzamentos industriais tem sido utilizada com esse objetivo. Pesquisas têm sido realizadas enfocando a qualidade de carne bovina nos últimos 20 anos, e a maciez é sempre apontada como fator essencial para o julgamento da qualidade do produto (Koohmaraie, 1994). Vários fatores influenciam a textura final da carne. Entre eles, destacam-se a maturidade (Culler et al., 1978), a quantidade e natureza química do colágeno (Bailey, 1972), a raça, a extensão da proteólise pós-abate (Dransfield, 1994), a velocidade de queda do $\mathrm{pH}$, a temperatura da carne no momento do rigor mortis (Marsh, 1977) e os métodos de preparo culinário (Apple et al., 1999).

O objetivo deste trabalho foi avaliar fatores que influenciam a textura da carne de novilhos Nelore e cruzados Limousin-Nelore.

\section{Material e Métodos}

O experimento foi realizado na Agropecuária Maragogipe, localizada em Itaquiraí, MS. Foram utilizados 50 novilhos castrados, sendo 25 da raça Nelore e 25 cruzados ( $1 / 2$ Limousin - $1 / 2$ Nelore), com idade entre 18 e 24 meses e peso médio de $340 \mathrm{~kg}$ no início do experimento. Os animais foram separados aleatoriamente em cinco grupos, compostos por cinco animais de cada grupo genético. Um dos grupos foi abatido no início do experimento e os demais permaneceram em sistema de criação em pasto, com suplementação nutricional, sendo abatidos de forma seriada ao longo de 204 dias, possibilitando a obtenção de animais em faixas crescentes de peso (Berg et al., 1978).

Os abates foram realizados no Frigorífico Bertin Ltda., em Naviraí, MS, de acordo com as normas e procedimentos oficiais (Brasil, 2002). Após o abate foram monitorados os valores de $\mathrm{pH}$ e temperatura do músculo longissimus dorsi, à altura da $12^{\underline{a}}$ costela, durante 24 horas, com o uso de potenciômetro de penetração com compensador e visor de temperatura, marca Mettler Toledo, modelo 1120-X. No momento da desossa, o músculo longissimus dorsi foi cortado transversalmente na altura da 12 a costela, o contorno do corte foi traçado em papel vegetal, para depois ser determinada a área de olho de lombo (AOL) em sistema analisador de imagens. Em seguida, foram retiradas quatro amostras de cerca de $2,5 \mathrm{~cm}$ de espessura cada, obtidas por cortes transversais do músculo, adjacentes à região da 12a costela. Essas amostras foram identificadas e embaladas a vácuo em sacos de polietileno. Três amostras foram congeladas imediatamente (carne fresca) no túnel de congelamento do frigorífico (cerca de $-30^{\circ} \mathrm{C}$ ) e uma foi destinada à maturação por 14 dias, à temperatura de $0-1^{\circ} \mathrm{C}$, e congelada após esse período. Em amostras frescas foram determinadas a composição centesimal, de acordo com AOAC International (1997); o comprimento do sarcômero (CS), conforme Heinemann et al. (2002), e o teor de colágeno total, pela quantificação de hidroxiprolina, segundo Kolar (1990). O teor de colágeno solúvel foi avaliado segundo Culler et al. (1978), com algumas modificações, em que uma alíquota de $5 \mathrm{~g}$ de carne finamente triturada foi homogeneizada com $20 \mathrm{~mL}$ de tampão fosfato $(0,1 \mathrm{M}$ a pH 7,0) em tubo de centrífuga, sob agitação. Os tubos permaneceram em banho-maria a $74^{\circ} \mathrm{C}$ por 20 minutos, com agitação a cada 30 segundos. Posteriormente, foram resfriados em banho de gelo até a temperatura ambiente e a suspensão foi centrifugada $(10.000 \mathrm{~g}, 15 \mathrm{minu}-$ tos). O teor de colágeno no precipitado foi determinado conforme Kolar (1990). A porcentagem de colágeno solúvel foi determinada descontando-se o teor da porção insolúvel do teor total de colágeno.

A maciez das amostras foi avaliada instrumentalmente pela determinação da força de cisalhamento em texturômetro $\mathrm{TAXT}_{2}$, equipado com célula de carne de Warner-Bratzler, padrão SMS (Stable Micro System). Duas amostras (uma fresca e uma maturada) foram descongeladas (estufa BOD a $4^{\circ} \mathrm{C}$ por 14 horas) e submetidas a cozimento em banhomaria $\left(100^{\circ} \mathrm{C}\right)$ até atingirem $70^{\circ} \mathrm{C}$ no interior, conforme descrito por Watanabe et al. (1996). Após serem resfriadas por 12 horas a $4^{\circ} \mathrm{C}$, foram retiradas pelo menos seis porções com $1 \mathrm{~cm}^{2}$ de área e aproximadamente $2,5 \mathrm{~cm}$ de comprimento, no sentido longitudinal em relação às fibras da amostra (Silva et al., 1999). Na avaliação, foi empregada a velocidade de cisalhamento de $5,0 \mathrm{~mm} / \mathrm{seg}$ e os resultados expressos como força máxima de cisalhamento, em quilogramas.

Foi utilizado delineamento experimental inteiramente casualizado, no esquema fatorial $2 \times 5$, com dois grupos genéticos (GG), Nelore e cruzados Limousin-Nelore e cinco faixas de peso ao abate (PA), com cinco repetições, cada animal representando uma unidade experimental. Foram 
determinadas as correlações simples entre as variáveis estudadas. As análises foram realizadas mediante o pacote estatístico SAS Institute (1989).

\section{Resultados e Discussão}

$\mathrm{O}$ pH final da carne dos animais dos dois últimos grupos abatidos, 24 horas após o abate, situou-se na faixa que Silva et al. (1999) consideram como “DFD moderado" (Figura 1). Esta é uma característica tecnológica causada por fatores estressantes préabate e pela conseqüiente redução das reservas de glicogênio muscular. Esse stress pré-abate pode ser atribuído ao desconforto térmico, uma vez que os abates ocorreram na época mais quente do ano.

Quando a temperatura do músculo atinge valores inferiores a $10^{\circ} \mathrm{C}$ antes da $10^{\underline{a}}$ hora após o abate, como se observou nas carcaças pertencentes aos grupos de abate 1 e 2 (Figura 2), observa-se a ocorrência de encurtamento pelo frio (Marsh, 1977; Felício, 1995). Segundo Judge et al. (1989), os fatores que mais influenciam a velocidade de resfriamento são o calor específico da carcaça, seu tamanho, quantidade externa de gordura e temperatura da câmara de estocagem. Considerando que as condições da câmara foram semelhantes em todos os abates, pode- se concluir que a redução da eficiência de dissipação de calor, causada pela maior cobertura de gordura e musculosidade dos animais dos últimos grupos de abate (Tabelas 1 e 2), foram responsáveis pela queda mais lenta de temperatura no interior da massa muscular. A observação visual dos perfis musculares evidenciou o efeito positivo do cruzamento sobre a conformação das carcaças. A evidência numérica desse efeito foi obtida com a determinação da AOL, que foi maior nas carcaças de animais cruzados $(\mathrm{P}<0,05)$, demonstrando a superioridade da massa muscular (Tabela 1). A AOL também apresentou uma clara tendência de aumento com o aumento do peso ao abate. Com relação à cobertura de gordura (CG), como a interação entre os tratamentos foi significativa, procedeu-se seu desdobramento (Tabela 2). Os animais Nelore iniciam com uma menor média ajustada de CG no PA1 $(\mathrm{P}<0,05)$ e essa diferença deixa de ser significativa nas maiores faixas de peso (PA4 e PA5). Houve uma maior taxa de deposição de gordura de cobertura desse grupo de animais, uma vez que iniciaram de menores valores e chegaram ao PA5 com maiores médias de CG, apresentando comportamento sempre crescente. As raças continentais européias têm como característica menor tendência à deposição de gordura. No caso específico da raça

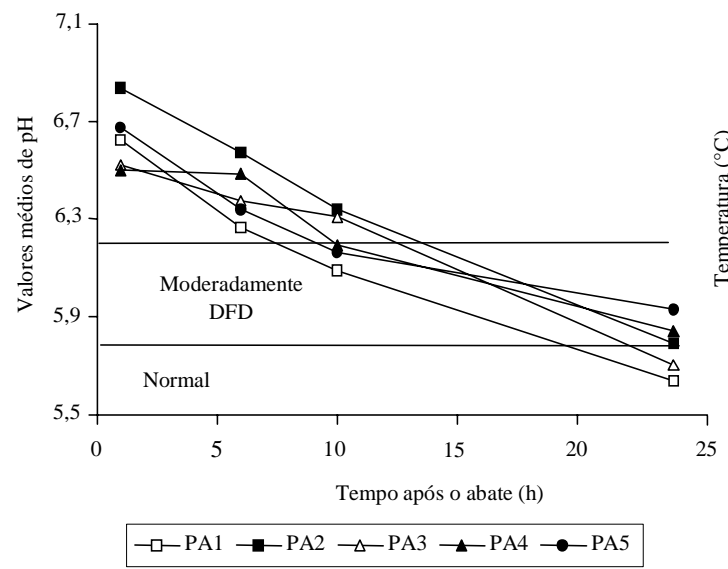

Figura 1. Curvas médias de queda de $\mathrm{pH}$ nas 24 horas após o abate nos diferentes grupos de abate. PA1, PA2, $\mathrm{PA} 3, \mathrm{PA} 4$ e PA5 correspondem às médias de peso vivo ao abate de 342,5 kg, 366,8 kg, 419,1 kg, 472,7 kg e 492,6 kg, respectivamente.

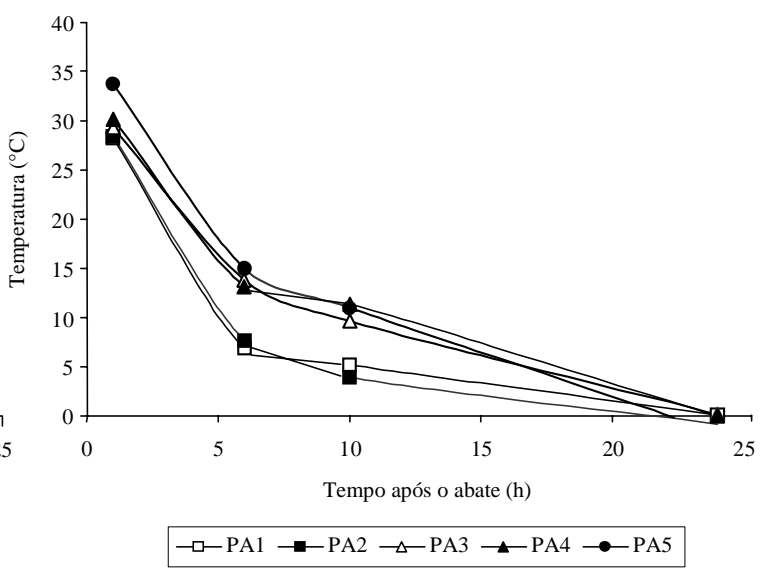

Figura 2. Curvas médias de queda de temperatura no longissimus dorsi nos grupos de abate. PA1, PA2, PA3, PA4 e PA5 correspondem às médias de peso vivo ao abate de $342,5 \mathrm{~kg}, 366,8 \mathrm{~kg}, 419,1 \mathrm{~kg}, 472,7 \mathrm{~kg}$ e $492,6 \mathrm{~kg}$, respectivamente.

Pesq. agropec. bras., Brasília, v. 38, n. 8, p. 963-971, ago. 2003 
Limousin, deve-se considerar que são animais de boa conformação muscular e peso final elevado. Assim, quando atingem peso de abate, ainda estão em fase de ganho de peso e de formação de tecido muscular (Lanna, 1997).

O grupo genético (GG) não influenciou significativamente o $C S(P>0,05)$, porém houve influência significativa $(\mathrm{P}<0,01)$ da faixa de peso ao abate nesse parâmetro (Tabela 1). Os animais dos grupos mais leves de abate apresentaram também menores valores de CS, o que pode contribuir de forma negativa na textura da carne pela maior compactação do tecido e conseqüente resistência ao cisalhamento.

Houve variação de força de cisalhamento $(\mathrm{FC})$ de 12,35 a $9,80 \mathrm{~kg}$, com diferença significativa $(\mathrm{P}<0,05)$ entre os grupos de animais de diferentes pesos de abate (Tabela 1). É importante observar que a lâmina da célula de Warner-Bratzler padrão SMS, utilizada neste trabalho, possui espessura de $3 \mathrm{~mm}$, ao passo que a espessura da lâmina padrão Warner-Bratzler é de 0,04 polegada, que equivale a aproximadamente $1,02 \mathrm{~mm}$. A espessura maior da lâmina certamente ele-

Tabela 1. Peso de carcaça quente (PCQ), área de olho de lombo (AOL), espessura de cobertura de gordura (CG), comprimento de sarcômero (CS), força de cisalhamento da carne fresca (FCF) e da carne maturada (FCM) em animais dos grupos genéticos (GG) Nelore $(\mathrm{N})$ e cruzados Limousin-Nelore (LN), em diferentes faixas de peso vivo ao abate $(\mathrm{PA})^{(1)}$.

\begin{tabular}{|c|c|c|c|c|c|c|}
\hline $\begin{array}{c}\text { Peso vivo ao } \\
\text { abate }^{(2)}\end{array}$ & PCQ (kg) & $\mathrm{AOL}\left(\mathrm{cm}^{2}\right)$ & $\mathrm{CG}(\mathrm{mm})$ & $\mathrm{CS}(\mu \mathrm{m})$ & $\mathrm{FCF}(\mathrm{kg})$ & $\mathrm{FCM}(\mathrm{kg})$ \\
\hline PA1 & $173,78 \mathrm{e}$ & $66,98 \mathrm{c}$ & 1,34 & $1,75 b c$ & $12,35 \mathrm{a}$ & $8,24 \mathrm{ab}$ \\
\hline PA2 & $189,56 \mathrm{~d}$ & $72,72 b c$ & 1,13 & $1,66 \mathrm{c}$ & $10,62 \mathrm{ab}$ & $8,66 a$ \\
\hline PA3 & $223,40 \mathrm{c}$ & $72,65 b c$ & 1,85 & $1,83 \mathrm{ab}$ & $10,94 \mathrm{ac}$ & $7,26 b$ \\
\hline PA4 & $246,86 b$ & $81,36 a$ & 2,60 & $1,79 \mathrm{abc}$ & $12,37 \mathrm{a}$ & $8,55 \mathrm{a}$ \\
\hline PA5 & $265,36 \mathrm{a}$ & $77,95 \mathrm{ab}$ & 2,50 & $1,92 \mathrm{a}$ & $9,80 \mathrm{~b}$ & $7,08 \mathrm{~b}$ \\
\hline Média de LN & $226,41 \mathrm{a}$ & $83,15 a$ & 1,92 & $1,76 \mathrm{a}$ & $10,50 \mathrm{~b}$ & $7,45 b$ \\
\hline Média de N & $213,18 b$ & $65,51 \mathrm{~b}$ & 1,84 & $1,82 \mathrm{a}$ & $11,85 \mathrm{a}$ & $8,50 \mathrm{a}$ \\
\hline $\begin{array}{l}\text { Fonte de } \\
\text { variação }\end{array}$ & \multicolumn{6}{|c|}{ Quadrado médio } \\
\hline PA & $1.4633,04 * *$ & $305,60 *$ & $4,40 * *$ & $0,09 * *$ & $12,01 *$ & $5,49^{*}$ \\
\hline GG & $2.188,04 * *$ & $3.891,56^{* *}$ & $0,07^{\mathrm{ns}}$ & $0,05^{\mathrm{ns}}$ & $22,65^{*}$ & $15,20 * *$ \\
\hline PA x GG & $177,86^{\mathrm{ns}}$ & $64,60^{\text {ns }}$ & $0,46^{*}$ & $0,01^{\mathrm{ns}}$ & $5,15^{\mathrm{ns}}$ & $2,08^{\mathrm{ns}}$ \\
\hline Resíduo & 102,40 & 75,13 & 0,15 & 0,02 & 3,73 & 1,56 \\
\hline $\mathrm{CV}(\%)$ & 4,60 & 11,66 & 20,78 & 8,12 & 17,28 & 15,71 \\
\hline
\end{tabular}

Tabela 2. Desdobramento da interação entre diferentes pesos ao abate (PA) e grupos genéticos (GG) do parâmetro espessura de cobertura de gordura $(\mathrm{CG})^{(1)}$.

\begin{tabular}{llllll}
\hline GG & PA1 & PA2 & PA3 & PA4 & PA5 \\
\hline Limousin-Nelore & $1,67 \mathrm{~cd}$ & $1,06 \mathrm{e}$ & $2,00 \mathrm{bc}$ & $2,60 \mathrm{a}$ & $2,27 \mathrm{ab}$ \\
Nelore & $1,00 \mathrm{e}$ & $1,20 \mathrm{de}$ & $1,70 \mathrm{~cd}$ & $2,60 \mathrm{a}$ & $2,73 \mathrm{a}$ \\
\hline
\end{tabular}

${ }^{(1)}$ Médias seguidas de letras distintas diferem entre si pelo teste de Duncan, a 5\% de probabilidade; PA1, PA2, PA3, PA4 e PA5 correspondem às médias de peso vivo ao abate de $342,5 \mathrm{~kg}, 366,8 \mathrm{~kg}, 419,1 \mathrm{~kg}, 472,7 \mathrm{~kg}$ e $492,6 \mathrm{~kg}$, respectivamente. 
va o valor da força máxima de cisalhamento. Embora os trabalhos científicos raramente especifiquem o padrão da célula de Warner-Bratzler utilizada, os resultados somente podem ser comparados quando o padrão da célula utilizada for o mesmo. $\mathrm{O}$ grupo mais leve (PA1) apresentou a maior média de FC e o PA5 a menor $(\mathrm{P}<0,05)$. Esses resultados sugerem tendência de decréscimo na força de cisalhamento com o aumento do peso ao abate dos animais, o que pode ser atribuído, principalmente, à menor contração do tecido, evidenciada pelo maior CS, com o avanço da faixa de peso dos animais, no intervalo estudado. Bouton et al. (1978) avaliaram a influência da idade de abate do animal na maciez da carne e observaram que os valores de FC, medida no músculo longissimus dorsi, foram reduzidos à metade com o aumento da idade do animal de nove para 42 meses, atribuindo esse efeito principalmente ao decréscimo da ocorrência de encurtamento pelo frio nas carcaças mais pesadas.

É interessante observar a característica multifatorial do atributo textura da carne. Com o aumento da idade do animal há tendência de redução da maciez, pelo aumento da estabilidade térmica do colágeno, resultante da formação de pontes cruzadas (Marsh, 1977). Considerando o longo tempo necessário para a renovação metabólica do colágeno (Bailey, 1972), pode-se concluir que, em animais jovens, a influência do tecido conjuntivo é pequena, prevalecendo o efeito de outros fatores, como a intensidade da proteólise e o grau de contração do tecido muscular.

O teor total de colágeno foi influenciado apenas pelo GG $(\mathrm{P}<0,05)$, com os animais cruzados apresentando média ligeiramente inferior aos animais Nelore $(\mathrm{P}<0,05)$ (Tabela 3). Os valores obtidos no estudo da solubilidade do colágeno foram influenciados somente pelo fator PA, sendo a média do PA1, de animais mais jovens e mais leves, significativamente maior $(\mathrm{P}<0,05)$ que as médias dos demais grupos, porém seu efeito não resultou em melhor textura da carne neste grupo de animais.

A maturação foi o fator que mais influenciou a textura da carne, promovendo redução de aproximadamente $30 \%$ na FC, em ambos os grupos genéticos

Tabela 3. Teor de umidade (U), proteína (P), gordura intramuscular (GI), cinzas (C), colágeno total (Col T) e solubilidade de colágeno (Sol C) do músculo longissimus dorsi de animais dos grupos genéticos (GG) Nelore (N) e cruzados Limousin-Nelore (LN), em diferentes faixas de peso ao abate (PA) ${ }^{(1)}$.

\begin{tabular}{|c|c|c|c|c|c|c|}
\hline $\begin{array}{c}\text { Peso vivo ao } \\
\text { abate }^{(2)}\end{array}$ & $\mathrm{U}(\%)$ & $\mathrm{P}(\%)$ & GI (\%) & $\mathrm{C}(\%)$ & $\mathrm{Col} \mathrm{T}(\%)$ & Sol C (\%) \\
\hline PA 1 & $76,03 \mathrm{ab}$ & $22,83 a$ & $0,64 \mathrm{c}$ & $1,03 \mathrm{a}$ & $0,48 \mathrm{a}$ & $21,62 \mathrm{a}$ \\
\hline PA 2 & $76,49 a$ & $21,58 b$ & $0,60 \mathrm{c}$ & $0,98 \mathrm{a}$ & $0,51 \mathrm{a}$ & $10,41 b$ \\
\hline PA 3 & $76,40 \mathrm{a}$ & $20,09 \mathrm{c}$ & $1,07 b c$ & $1,02 \mathrm{a}$ & $0,52 \mathrm{a}$ & $13,07 b$ \\
\hline PA 4 & $75,07 \mathrm{c}$ & $20,89 b c$ & $1,53 \mathrm{ab}$ & $1,01 \mathrm{a}$ & $0,50 \mathrm{a}$ & $11,71 \mathrm{~b}$ \\
\hline PA 5 & $75,38 \mathrm{bc}$ & $20,90 \mathrm{bc}$ & $1,76 \mathrm{a}$ & $1,05 \mathrm{a}$ & $0,49 \mathrm{a}$ & $13,21 \mathrm{~b}$ \\
\hline Média de LN & $75,79 a$ & $21,63 a$ & $1,08 \mathrm{a}$ & $1,02 \mathrm{a}$ & $0,48 b$ & $13,80 \mathrm{a}$ \\
\hline Média de N & $75,96 \mathrm{a}$ & $20,89 \mathrm{~b}$ & $1,19 \mathrm{a}$ & $1,02 \mathrm{a}$ & $0,52 \mathrm{a}$ & $14,04 \mathrm{a}$ \\
\hline $\begin{array}{l}\text { Fonte de } \\
\text { variação }\end{array}$ & \multicolumn{6}{|c|}{ Quadrado médio } \\
\hline PA & $3,91 * *$ & $10,51 * *$ & $2,45^{* *}$ & $0,01^{\mathrm{ns}}$ & $0,0022^{\mathrm{ns}}$ & $182,24 * *$ \\
\hline GG & $0,37^{\mathrm{ns}}$ & $6,93 *$ & $0,418^{\text {ns }}$ & $0,00^{\mathrm{ns}}$ & $0,0202 *$ & $1,98^{\mathrm{ns}}$ \\
\hline PA x GG & $0,98^{\mathrm{ns}}$ & $2,41^{\mathrm{ns}}$ & $0,35^{\mathrm{ns}}$ & $0,01^{\mathrm{ns}}$ & $0,0023^{\text {ns }}$ & $18,40^{\mathrm{ns}}$ \\
\hline Resíduo & 0,61 & 1,13 & 0,24 & 0,01 & 0,0045 & 34,66 \\
\hline $\mathrm{CV}(\%)$ & 1,03 & 5,00 & 43,27 & 7,90 & 13,43 & 42,32 \\
\hline
\end{tabular}

${ }^{(1)}$ Em cada coluna, médias seguidas da mesma letra não diferem entre si pelo teste de Duncan, a 5\% de probabilidade. (2)PA1, PA2, PA3, PA4 e PA5

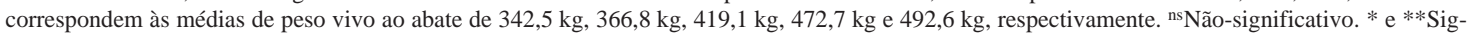
nificativo a $5 \%$ e a $1 \%$ de probabilidade, respectivamente. 
avaliados. Além disso, a maturação promoveu redução na variação da FC das amostras, com valores de 8,66 a $7,08 \mathrm{~kg}$. Somente o último grupo abatido teve força de cisalhamento da carne maturada (FCM) significativamente menor $(\mathrm{P}<0,05)$ que os demais grupos, apresentando, portanto, carne mais macia.

Puga et al. (1999) relataram aumento de maciez de $20 \%$ com a maturação por 14 dias. Já Rubensan et al. (1998) observaram efeito ainda mais pronunciado em carnes maturadas por 10 dias, com redução de FC de 6,10 kg para 3,67 kg em animais Polled Hereford e de $8,12 \mathrm{~kg}$ para $5,00 \mathrm{~kg}$ em animais $5 / 8$ Hereford $3 / 8$ Nelore.

O GG influenciou os valores de FC e FCM e, em ambos os parâmetros, os animais cruzados apresentaram menores médias $(\mathrm{P}<0,05)$ que os animais Nelore, portanto, com carne mais macia. Já está bem estabelecida a associação do genótipo Bos indicus com a redução de maciez da carne, o que pode ser atribuído a diversos fatores. Os animais zebuínos são geralmente criados em regime extensivo e abatidos com idade mais avançada, o que pode influenciar o estado químico das fibras de colágeno, afetando negativamente a textura. Além disso, apresentam baixa tendência de deposição de gordura e massa muscular geralmente inferior à de raças européias de corte, o que pode favorecer o rápido resfriamento da carcaça e aumentar o grau de contração do tecido muscular na refrigeração (Marsh, 1977). Considerando que, neste trabalho, os animais dos dois grupos genéticos apresentavam idade semelhante e que o CS não diferiu significativamente entre eles, não se pode atribuir a diferença observada na FC a esses fatores. Pringle et al. (1997) observaram valores em torno de 7,5 kg e 6,1 kg, respectivamente, na FC de carne fresca e maturada por 14 dias, em animais $100 \%$ Brahman, e os valores decresceram com o aumento da participação do sangue Angus. Whipple et al. (1990) atribuem esse efeito de melhora da textura com a menor participação do genótipo Bos indicus a uma menor atividade de calpastatina, inibidor natural das enzimas calpaínas, em bovinos Bos taurus. Oliveira (2000) conclui que, entre os vários fatores que influenciam a diferença na maciez da carne fresca de animais Bos indicus e Bos taurus, o mais importante é a extensão da proteólise por enzimas endógenas sofrida pelo tecido muscular após o abate. Embora esse fator não tenha sido avaliado diretamente neste trabalho, observou-se aumento da significância da influência do GG na FC após a maturação (Tabela 1), evidenciando a importância da proteólise endógena nesse resultado.

As médias do teor de cinzas não apresentaram diferença significativa $(\mathrm{P}>0,05)$ com relação ao $\mathrm{PA}$ nem ao GG, representando sempre cerca de $1 \%$ da composição da carne (Tabela 3). Os teores de gordura intramuscular variaram significativamente $(\mathrm{P}<0,01)$ com o PA, mas não foram influenciados pelo GG (P>0,05). Dentro do intervalo de peso ao abate utilizado, todos os animais apresentaram baixo teor de gordura intramuscular no longissimus dorsi, o que pode ser constatado pelos valores de $1,58 \%$ e $1,76 \%$, respectivamente, nas amostras provenientes dos animais das faixas quatro e cinco. Abularach et al. (1998), estudando a qualidade de contrafilé de touros jovens Nelore, reportaram teores de gordura em torno de 1,71\% em animais com cerca de $497 \mathrm{~kg}$ de peso ao abate. Estes autores também relataram que os teores de gordura foram menores que os encontrados na literatura, o que pode prejudicar a suculência e percepção de maciez, principalmente se a carne for preparada pela cocção em calor seco. Segundo Campion et al. (1975), é desejável que a carne apresente teor de gordura intramuscular superior a $2 \%$, para favorecer as características de qualidade. As médias de gordura no músculo longissimus dorsi foram inferiores a esse valor, o que pode ter contribuído de forma negativa na textura da carne.

Houve pequeno decréscimo no teor de umidade com o aumento do peso ao abate e oscilações nos teores de proteína (Tabela 3 ), que podem ser atribuídas às oscilações nos demais componentes.

Em ambos os grupos genéticos, a CG e a gordura intramuscular (GI) aumentaram significativamente com o aumento do peso da carcaça quente (Tabela 4). A AOL teve correlação maior e mais significativa com o peso de carcaça quente (PCQ) nas amostras provenientes de animais cruzados do que de animais Nelore, demonstrando a tendência do cruzamento de aumentar a musculosidade da carcaça. Essa maior área de secção transversal do longissimus dorsi promoveu uma proteção contra a dissipação do calor no interior do músculo. A temperatura 
Tabela 4. Coeficientes de correlação linear entre as variáveis peso de carcaça quente (PCQ), teor de gordura intramuscular (GI), espessura de cobertura de gordura (CG), área de olho de lombo (AOL), temperatura após 10 horas de resfriamento (T10), pH após 24 horas do abate ( $\mathrm{pH} 24$ ), comprimento de sarcômero (CS), solubilidade de colágeno (Sol C), forca de cisalhamento da carne fresca (FCF), força de cisalhamento da carne maturada (FCM) em animais Nelore e cruzados Limousin-Nelore ${ }^{(1)}$.

\begin{tabular}{|c|c|c|c|c|c|c|c|c|c|}
\hline Variáveis & GI & CG & $\mathrm{AOL}$ & $\mathrm{T} 10$ & $\mathrm{pH} 24$ & $\mathrm{CS}$ & Sol C & FCF & FCM \\
\hline & & & & & Nelore & & & & \\
\hline PCQ & $0,76^{* *}$ & $0,77 * *$ & $0,47 *$ & $0,87 * *$ & $0,78 * *$ & 0,24 & $-0,22$ & $-0,34$ & $-0,32$ \\
\hline GI & & $0,74 * *$ & 0,40 & $0,71 * *$ & $0,53 * *$ & 0,07 & 0,01 & $-0,21$ & $-0,12$ \\
\hline $\mathrm{CG}$ & & & 0,35 & $0,77 * *$ & $0,64 * *$ & 0,40 & $-0,07$ & $-0,43^{*}$ & $-0,19$ \\
\hline $\mathrm{AOL}$ & & & & 0,33 & $0,51 *$ & 0,33 & $-0,24$ & $-0,49 *$ & 0,19 \\
\hline $\mathrm{T} 10$ & & & & & $0,65 * *$ & 0,38 & $-0,03$ & $-0,28$ & $-0,36$ \\
\hline pH 24 & & & & & & 0,39 & $-0,30$ & $-0,58 * *$ & $-0,38$ \\
\hline $\mathrm{CS}$ & & & & & & & 0,15 & $-0,44$ & $-0,21$ \\
\hline Sol C & & & & & & & & 0,02 & 0,13 \\
\hline \multirow[t]{2}{*}{$\mathrm{FCF}$} & & & & & & & & & 0,12 \\
\hline & \multicolumn{9}{|c|}{ Cruzado (1/2 Limousin e $1 / 2$ Nelore) } \\
\hline PCQ & $0,71 * *$ & $0,67 * *$ & $0,58 * *$ & $0,78 * *$ & $0,51 * *$ & $0,54 * *$ & $-0,58 * *$ & $-0,26$ & $-0,25$ \\
\hline GI & & $0,51 *$ & 0,26 & $0,65 * *$ & 0,38 & 0,32 & $-0,59 * *$ & $-0,07$ & $-0,10$ \\
\hline CG & & & 0,42 & $0,77 * *$ & 0,17 & 0,25 & $-0,29$ & 0,27 & 0,09 \\
\hline $\mathrm{AOL}$ & & & & $0,60 * *$ & 0,27 & 0,36 & $-0,31$ & $-0,19$ & 0,09 \\
\hline $\mathrm{T} 10$ & & & & & 0,34 & $0,50 *$ & $-0,34$ & $-0,12$ & $-0,10$ \\
\hline $\mathrm{pH} 24$ & & & & & & 0,05 & $-0,39$ & $-0,09$ & 0,19 \\
\hline CS & & & & & & & $-0,20$ & $-0,49 *$ & $-0,42 *$ \\
\hline Sol C & & & & & & & & 0,26 & 0,20 \\
\hline FCF & & & & & & & & & $0,46^{*}$ \\
\hline
\end{tabular}

$* \mathrm{e} * *$ Significativo a $5 \%$ e a $1 \%$ de probabilidade, respectivamente, pelo teste $\mathrm{F}$.

10 horas após o abate foi influenciada pela gordura de cobertura, pela GI e pelo PCQ, em ambos os grupos raciais, mas a AOL influenciou a queda de temperatura apenas nas carcaças de animais cruzados. Nas carcaças de animais cruzados, a temperatura à $10^{\mathrm{a}}$ hora apresentou correlação com o CS, que, por sua vez, apresentou correlação negativa tanto com a força de cisalhamento da carne fresca $(\mathrm{FCF})$ quanto com a da carne maturada. Verificou-se correlação negativa da solubilidade do colágeno com o PCQ e com a gordura intramuscular. $\mathrm{O}$ aumento de peso e gordura corresponde a maior tempo de vida, o que poderia explicar essa correlação. A correlação entre a FC e GC e a AOL nos animais Nelore foi negativa. Isso é atribuído ao efeito de proteção desses fatores contra o resfriamento excessivamente rápido, prevenindo o encurtamento pelo frio e, portanto, melho- rando a textura da carne. Também se verificou, nesse grupo, correlação negativa da FCF com o pH, 24 horas após o abate. Carnes com pH mais elevado apresentam maior capacidade de retenção de água e menor compactação do tecido muscular, por estarem acima do valor de $\mathrm{pH}$ correspondente ao ponto isoelétrico da maioria das proteínas da carne (Judge, 1989). O efeito do CS na textura da carne ficou demonstrado apenas no grupo de animais cruzados, em que se verificou correlação negativa entre FCF e FCM com este parâmetro.

\section{Conclusões}

1. Os novilhos cruzados Limousin-Nelore apresentam maior maciez de carne que novilhos Nelore.

2. A maturação por 14 dias é importante contribuinte na maciez da carne bovina, agindo positivamen- 
te na textura quando comparada à maciez de carne não maturada.

3. A textura final da carne é influenciada pelo grupo genético e, principalmente, por fatores que determinam a velocidade de resfriamento da carcaça.

\section{Agradecimentos}

À Fapesp, pelo apoio financeiro; à Associação Brasileira de Criadores de Limousin e à Agropecuária Maragogipe, que disponibilizaram os animais e viabilizaram sua criação até o abate; à Navimix, responsável pelo suplemento nutricional fornecido aos animais; ao Frigorífico Bertin Ltda., que disponibilizou as instalações e recursos humanos necessários ao abate experimental, além das amostras utilizadas no experimento.

\section{Referências}

ABULARACH, M. L. S.; ROCHA, C. E.; FELÍCIO, P. E. Características de qualidade do contra filé (m. L. dorsi) de touros jovens da raça Nelore. Ciência e Tecnologia de Alimentos, Campinas, v. 18, n. 2, p. 205-210, 1998.

ANUALPEC 2002: anuário da pecuária brasileira. São Paulo: FNP Consultoria e Comércio/Argos, 2002. 400 p.

AOAC INTERNATIONAL (Gaithersburg, Estados Unidos). Official methods of analysis of the AOAC International. $16^{\text {th }}$ ed. Gaithersburg, 1997. v. 1.

APPLE, J. K.; RAKES, L. K.; WATSON, H. B. Cooking and shearing methodology effects on Warner-Bratzler shear force values of pork. Journal of Muscles Foods, Trumbull, v. 10 , p. $269-277,1999$.

BAILEY, A. J. The basis of meat texture. Journal of the Science of Food and Agriculture, Chichester, v. 23, p. 995-1107, 1972.

BERG, R. T.; ANDERSEN, B. B.; LIBORIUSSEN, T. Growth of bovine tissues - 1: genetic influence on growth patterns of muscle, fat and bone in young bulls. Animal Production, Edinburgh, v. 26, p. 245-258, 1978.

BOUTON, P. E.; FORD, A. L.; HARRIS, P. V.; SHORTHOSE, W. R.; RATCLIFF, D.; MORGAN, J. H. L. Influence of animal age on the tenderness of beef: muscle differences. Meat Science, Barking, v. 2, n. 4 p. 301-311, 1978.
BRASIL. Ministério da Agricultura. Decreto-lei nº 2244, de 4 de junho de 1997. Regulamento da Inspeção Industrial e Sanitária de Produtos de Origem Animal. Brasília, 1997. Disponível em: <www.agricultura.gov.br/sda/dipoa/ republport210.html>. Acesso em: 29 nov. 2002.

CAMPION, D.R.; CROUSE, J.D.; DIKEMAN, M.E. Predictive value of USDA beef quality grade factors for cooked meat palatability. Journal of Food Science, Albany, v. 40, p.1225-1228, 1975.

CULLER, R. D.; PARRISH JUNIOR, F. C.; SMITH, G. C.; CROSS, H. R. Relationship of myofibril fragmentation index to certain chemical, physical and sensory characteristics of bovine longissimus muscle. Journal of Food Science, Champaign, v. 43, p. 1177-1180, 1978.

DRANSFIELD, E. Optimization of tenderization, ageing and tenderness. Meat Science, Barking, v. 36, p. 105$121,1994$.

FELÍCIO, P.E. Maciez da carne: fator de competitividade. DBO Rural, São Paulo, v. 13, n. 17A, p. 88-91, fev. 1995. Especial pecuária de corte.

HEINEMANN, R. J. B.; PINTO, M. F.; PONSANO, E. H. G.; PERRI, S. H. V. Método simples para estimar encurtamento pelo frio em carne bovina. Ciência Rural, Santa Maria, v. 32, n. 2, p. 335-339, 2002.

IBGE (Rio de Janeiro, RJ). Pesquisa pecuária municipal. Disponível em: <www.sidra.ibge.gov.br/bda/pecua > Acesso em: 20 set. 2002.

JUDGE, M. D.; ABERLE, E. D.; FORREST, J. C.; HEDRICK, H. B.; MERKEL, R. A. Principles of meat science. $2^{\text {nd }}$ ed. Dubuque: Kendall Hunt, 1989. 351 p.

KOLAR, K. Colorimetric determination of hidroxiproline as measure of collagen content in meat and meat products: NMK collaborative study. Journal of the Association of Official Analytical Chemists, Washington, v. 73, p. 54-57, 1990.

KOOHMARAIE, M. Muscle proteinases and meat aging. Meat Science, Barking, v. 36, p. 93-104, 1994.

LANNA, D. P. Fatores condicionantes e predisponentes da puberdade e da idade de abate. In: SIMPÓSIO SOBRE PECUÁRIA DE CORTE, 4., 1996, Piracicaba. Anais... Piracicaba: Fealq, 1997. p. 79-97.

MARSH, B. B. The basis of quality in muscle foods: symposium the basis of tenderness in muscle foods. Journal of Food Science, Champaign, v. 42, n. 2, p. 295297, 1977. 
OLIVEIRA, A. L. Maciez da carne bovina. Cadernos Técnicos de Veterinária e Zootecnia, Belo Horizonte, n. 33, p. 7-18, 2000.

PRINGLE, T. D.; WILLIAMS, S. E.; LAMB, B. S.; JOHNSON, D. D.; WEST, R. L. Carcass characteristics, the calpain proteinase system, and aged tenderness of Angus and Brahman crossbred steers. Journal of Animal Science, Champaign, v. 75, p. 2955-2961, 1997.

PUGA, D. M. U.; CONTRERAS, C. J. C.; TURNBULL, M. R. Avaliação do amaciamento da carne bovina de dianteiro (Triceps brachi) pelos métodos de maturação, estimulação elétrica, injeção de ácidos e tenderização mecânica. Ciência e Tecnologia de Alimentos, Campinas, v. 19, n. 1, p. 88-96, 1999.

RUBENSAM, J. M.; FELÍCIO, P. E.; TERMIGNONI, C. Influência do genótipo Bos indicus na atividade de calpastatina e na textura da carne de novilhos abatidos no sul do Brasil. Ciência e Tecnologia de Alimentos, Campinas, v. 18, n. 4, p. 405-409, 1998.

SAS INSTITUTE (Cary, Estados Unidos). SAS user's guide: statistics: version $6.4^{\text {th }}$ ed. Cary, $1989.846 \mathrm{p}$.

SILVA, J. A.; PATARATA, L.; MARTINS, C. Influence of ultimate $\mathrm{pH}$ on bovine meat tenderness during ageing. Meat Science, Barking, v. 52, p. 453-459, 1999.

WATANABE, A.; DALY, C. C.; DEVINE, C. E. The effects of ultimate $\mathrm{pH}$ of meat on tenderness changes during ageing. Meat Science, Barking, v. 42, n. 1, p. 67-78, 1996.

WHIPPLE, G.; KOOHMARAIE, M.; DIKEMAN, M. E.; CROUSE, J. D.; HUNT, M. C.; KLEMM, R. D. Evaluation of attributes that affect Longissimus muscle tenderness in Bos taurus and Bos indicus cattle. Journal of Animal Science, Champaign, v. 68, p. 2716-2728, 1990. 